\title{
Mitteilungen der ÖGKM
}

J. Miner. Stoffwechs. Muskuloskelet. Erkrank. 2021 - 28:25 https://doi.org/10.1007/s41970-021-00148-8

(c) Springer-Verlag GmbH Austria, ein Teil von Springer Nature 2021

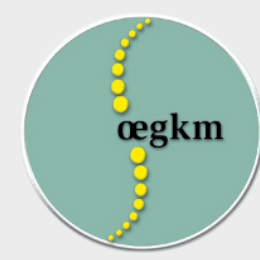

Präsident

ao.Univ.-Prof. Dr. Hans Peter Dimai (V.i.S.d.P.)

Schatzmeisterin

Ao. Univ.-Prof. Dr. Katharina Kerschan-Schindl

Generalsekretär

Priv.-Doz. Dr. Christian Muschitz

E-Mail: office@oegkm.at

Homepage: www.oegkm.at
Liebe Kolleginnen und Kollegen!

\section{Osteoporoseforum 2021}

Aufgrund der nach wie vor angespannten Covid-Situation in Österreich hat der Vorstand beschlossen, das Osteoporoseforum 2021 auf folgenden Termin zu verschieben:

14.-16. Oktober 2021 St. Wolfgang/Wolfgangsee

Dieser Kongress ist als Präsenzkongress geplant.

\section{Projektpreis der ÖGKM}

Die ÖGKM schreibt für 2020/2021 drei mit jeweils maximal Euro 5000,00 dotierte Projektpreise aus. Dadurch soll die wissenschaftliche Arbeit auf dem Gebiet des Knochen- und Mineralstoffwechsels gefördert werden. Weiterführende Informationen finden Sie auf der Homepage: www.oegkm.at

\section{Leitlinien}

- Die gemeinsame Leitlinie der ÖGKM mit der ÖGP (Österreichische Gesellschaft für Pneumologie) ist in der Phase der Finalisierung. Die Veröffentlichung ist bis Sommer 2021 geplant.

- Eine gemeinsame Leitlinie der ÖGKM in Kooperation der Österr. Gesellschaft für Intensivmedizin wird derzeit erstellt.

Österreichische Frakturdaten FRAX Update

Derzeit werden die Frakturdaten mit einer neuen Risikostratifizierung berechnet - Updates folgen.

Herzliche Grüße,

\section{Ihr Christian Muschitz}

Hier steht eine Anzeige. 黛 Springer 